Proceedings of the International School and Conference on Optics and Optical Materials, ISCOM07, Belgrade, Serbia, September 3-7, 2007

\title{
A Comparative Analysis of Associative Properties of Fourier versus Walsh Digital Holograms
}

\author{
A. DerzhypolskyY ${ }^{a, *}$ D. Melenevskyy ${ }^{b}$
}

\author{
AND A. GNATOVSKYY ${ }^{b}$
}

${ }^{a}$ Kyiv National Taras Shevchenko University, Kyiv, Ukraine

${ }^{b}$ Institute of Physics of NASU, Kyiv, Ukraine

A digital implementation of the Fourier holography scheme with identical reference and signal beams is considered. Investigations of the properties of this scheme were reported on in a number of papers. At the same time a concept of virtual optics is widely used in data encryption and data processing, whereas digital implementation of the Fourier holography scheme may be provided using not only discrete Fourier transform. Another discrete digital transform may be used as soon as it has several properties similar to the Fourier transform. The Walsh transform is the subject to consider in this work. A comparison of digital Fourier and Walsh versions of the named holographic scheme is conducted by means of their associative properties. Considered is the retrieving of information stored in the hologram using parts of different amounts of the original data. Comparison is provided with the two types of data stored: a raster image representing visual information and an image representing a set of data bits. Comparable parameters are the mean image contrast for visual data image and the bit detection accuracy for a set of data bits.

PACS numbers: 42.30.Kq, 42.30.Va, 42.30.Sy, 42.40.--i

\section{Introduction}

In recent years the investigations in holography concerning the issues of correlators, data processing and encryption etc. have been more and more becoming rather of simulation than of real experiments. Such a shift in research methods comes, on the one hand, from the development of analytical and computational apparatus of Fourier holography, and, on the other hand, from well studied and well predictable properties of Fourier holograms. In many cases there is conceptually no need to switch from simulation to a real experiment. This is a so-called concept of virtual optics (VO) [1, 2]. The VO means the implementation of data processing or encrypting algorithms based on certain optical setup and involving corresponding data transformations. Almost always the kernel of those transformations is

*corresponding author; e-mail: shaman@univ.kiev.ua 
either Fourier transform (FT) [1] or fractional Fourier transform (FRFT) [3, 4]. However, abstracting from optical fundamentals of VO, any other transform may be used as a kernel as soon as it has certain properties similar to FT. Probably, the use of some kernel more specific to digital data than FT can produce more effective VO algorithm.

As shown in [5], a concept of logical holography in the domain of lattice functions may be built up on the base of Walsh transform (WT). WT is fundamentally discrete as against to discrete FT which is only the sampled version of fundamentally continuous FT. In this paper we consider consequences of such kernel change from FT to WT in VO algorithm based on Fourier-holography scheme with identical reference and signal beams. This scheme is known mainly for the capability of reconstruction of stored image dependent on recognition of the part of that image, in other words the associative image reconstruction $[6,7]$.

\section{Optical setup and fundamentals}

The original optical setup of Fourier holography with identical reference and signal beams is shown in Fig. 1. The laser beam from laser L comes through the beam expander $\mathrm{BE}$ to the transparency $\mathrm{T}$ (with target image) coupled with the random phase modulator $\mathrm{D}$ (the diffuser). Then, split by beam splitter BS, it produces the two identical beams 1 and 2 . The beams are brought together on the hologram $\mathrm{H}$ in the focal plane of the first FT-objective $\mathrm{O}_{1}$ with the help of deflecting mirror BD. Actually, the beams are not strictly brought together. A slight misalignment introduced by the mirror BD is needed for the proper operation of the setup. At that the hologram is recorded. The restoration of the hologram is carried out with partially masked (to the different extent) transparency $\mathrm{T}$ and blocked beam 1. The beam restored in +1 diffraction order comes through the second FT-objective to form the image on the CCD array of camera C, connected to computer PC, where the restored image may be observed and saved.

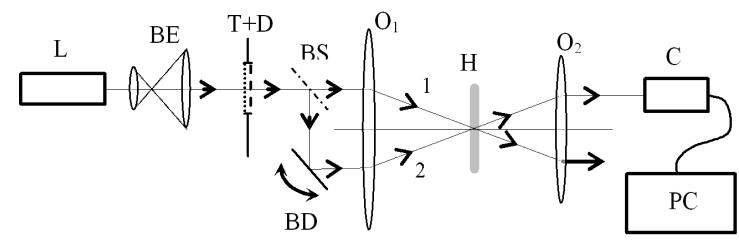

Fig. 1. Fourier holography with identical reference and signal beams, the original setup. $\mathrm{L}$ - laser; BE - beam expander; $\mathrm{T}+\mathrm{D}$ - transparency image coupled with the diffuser; $\mathrm{BS}$ - beam splitter; $\mathrm{BD}$ - beam deflector; $\mathrm{O}_{1}, \mathrm{O}_{2}$ - Fourier-transform objectives; $\mathrm{H}$ - hologram; C - CCD camera; PC - computer.

The mathematics of the operation of the scheme is the following. The complex amplitude of the wave restored in +1 diffraction order in the plane just after the hologram is described as [7]: 


$$
R_{+1}=A^{*} A_{\mathrm{s}} A_{\mathrm{p}}
$$

and in the plane of CCD-array

$$
r_{+1}=F^{-1}\left\{R_{+1}\right\}=\left(a_{\mathrm{s}} \otimes a\right) * a_{\mathrm{p}} \equiv\left(a_{\mathrm{p}} \otimes a\right) * a_{\mathrm{s}} .
$$

In the above formulas $a$ and $a_{\mathrm{p}}$ denote complex amplitude of the wave passed through the transparency coupled with the diffuser, unmasked and partially masked accordingly, $a_{\mathrm{s}}$ denotes the complex amplitude of the wave corresponding to the beam 2. $A$ and $A_{\mathrm{p}}$ are the FTs of the $a$ and $a_{\mathrm{p}}$, accordingly. $A_{\mathrm{s}}$ is the FT of $a_{\mathrm{s}}$ - the same as $A$, but slightly shifted in the hologram plane. $F^{-1}\{\ldots\}$ is the inverse FT operator, ${ }^{*}$ is the complex conjugation, $\otimes$ and $*$ are the correlation and convolution operators, accordingly. The two convolution-correlation notations given in formula (2) are possible due to commutative property of multiplication in the Fourier domain.

The part of the original image $a_{\mathrm{p}}$ is recognized in the correlation $a_{\mathrm{p}} \otimes a$ (see (2), the second notation) thus producing $\delta$-like function which subsequently produces the image close to original by means of convolution with $a_{\mathrm{s}}$. On the other hand, the possibility of producing $\delta$-like function by $a_{\mathrm{s}} \otimes a$ and subsequently restoring the image close to $a_{\mathrm{p}}$ is suppressed by the shift in Fourier domain introduced to $a_{\mathrm{s}}$ by the mirror $\mathrm{M}$ as mentioned above. Thus the restoration of the image close to the original occurs as soon as $a_{\mathrm{p}}$ is the part of that original image.

\section{Computational fundamentals}

In this section we describe some basics and some important peculiarities of VO computations for this scheme due to the properties of both FT and WT. Everywhere here we consider two-dimensional case.

\subsection{Discrete Fourier transform}

By definition the discrete FT (DFT) $A_{k l}$ of the lattice function $a_{m n}$ given for $n=0, \ldots, N-1, m=0, \ldots, M-1$ is

$$
A_{k l}=\sum_{m, n} a_{m n} \omega_{M}^{m k} \omega_{N}^{n l}
$$

where $\omega_{M}=\sqrt[M]{-1}=\mathrm{e}^{-2 \pi \mathrm{i} / M}, k=0, \ldots, M-1, l=0, \ldots, N-1$, or in matrix form

$$
\boldsymbol{A}=\boldsymbol{F}_{M} \boldsymbol{a} \boldsymbol{F}_{N}
$$

where the matrices $\boldsymbol{A}, \boldsymbol{a}, \boldsymbol{F}_{M}, \boldsymbol{F}_{N}$ are of sizes of $M \times N, M \times N, M \times M$ and $N \times N$ with elements of $A_{k l}, a_{m n}, \omega_{M}^{m k}$ and $\omega_{N}^{m k}$, accordingly. The rows and columns of transform matrices $\boldsymbol{F}_{M}, \boldsymbol{F}_{N}$ are sampled complex exponential functions.

In VO calculations involving multiplication in the Fourier domain (convolution or correlation in image domain) at least twice larger matrices than the processed image itself should be used to produce the result consistent with real experiment. This is because of expansion of the result of convolution (correlation) due to partial overlapping of convolved functions. In the case of DFT if such an expansion appears larger than the matrix used it wraps inside at the borders and 
introduces an additional noise to the output. Ideally the matrix should be the larger the more multiplications in FT domain are involved. But in practice it is quite enough to use the matrix just twice larger than the image itself.

Shift in FT domain introduced in $A_{\mathrm{s}}(1)$ is, of course, discrete in DFT VO calculations and is represented by element-wise circular shift of the corresponding matrix.

\subsection{Walsh transform}

Walsh transform $A_{k l}$ of the lattice function $a_{m n}$ given for $n=0, \ldots$, $N-1, m=0, \ldots, M-1$ in the matrix form is defined as

$$
\boldsymbol{A}=\boldsymbol{H}_{M} \boldsymbol{a} \boldsymbol{H}_{N},
$$

where $\boldsymbol{H}_{M}$ and $\boldsymbol{H}_{N}$ are the Hadamard matrices of size of $M \times M$ and $N \times N$, accordingly. The rows and the columns of Hadamard matrices are the Walsh functions. The basic definition of Hadamard matrices is the following [8]:

$$
H_{1}=[1], \quad H_{2 N}=\left[\begin{array}{cc}
H_{N} & H_{N} \\
H_{N} & -H_{N}
\end{array}\right], \quad N=2^{n} .
$$

There are also another definitions with $N$ other than power of 2 but those are not commonly used.

Analogously to FT the multiplication in WT domain corresponds to convolution in image domain. However, in this case the convolution is quite different than in FT, it is the logical convolution and it has another definition in image domain. The most important property of logical convolution as for the purpose of this work is that its result is not expanded and has the same size as the convolved functions. Hence there is no need to use matrices larger than the image itself.

Another important in VO peculiarity of WT against FT is that the Hadamard matrices are real while the FT transform matrices are complex. Thereby for real functions convolution and correlation that only differ by complex conjugation in FT domain are indistinguishable in WT domain. That is why only the complex diffuser (coupled with the transparency in the scheme in Fig. 1) should be used in WT VO calculations, whereas in FT calculations the binary diffuser with transmittance +1 and -1 may be successfully used.

The last but not the least important peculiarity of WT implementation of the considered $\mathrm{VO}$ scheme is the representation of the shift needed to be introduced in $A_{\mathrm{s}}(1)$. The shift in WT domain is represented as the logical shift of the corresponding matrix [5].

\section{Computational experiment}

The VO computational experiments with both FT and WT were carried out in MatLab 6.5R13. The images were of size of $512 \times 512$ pixels. The diffuser was represented as a matrix of complex numbers $\mathrm{e}^{\mathrm{i} \phi}$ with argument $\phi$ being a random number with uniform distribution in range $[0,2 \pi]$. FT was performed by the built-in MatLab function $f f t 2$ with zero-padding the image to the size of $1024 \times 1024$. 

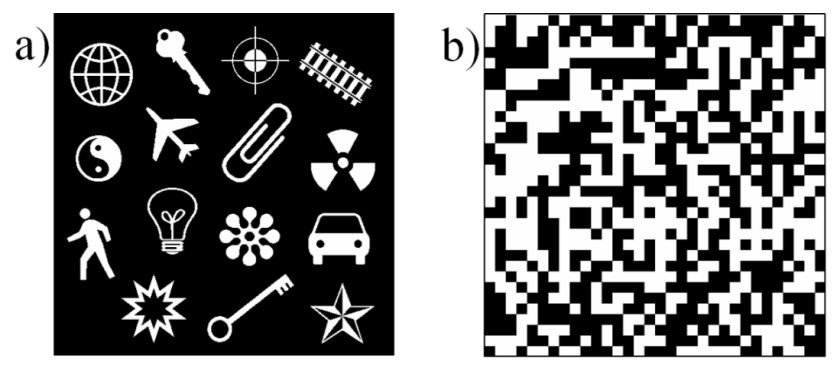

Fig. 2. The images used in contrast test (a) and bit detection test (b).

WT was performed following the formula (5) with Hadamard matrix produced following the definition (6). The shift was horizontal with value of 32 pixels.

The two different images were used to conduct the two types of the experiments. In both experiments there was studied the quality of the image restored by VO scheme with different level of masking of restoring image. The first experiment was performed with black-and-white image containing several objects and representing some visual information (Fig. 2a). The measured parameter here was the average contrast in restored image

$$
V=\frac{I_{\max }-I_{\min }}{I_{\max }+I_{\min }}
$$

$I_{\max }$ and $I_{\min }$ are the average intensities of the areas of restored image corresponding to black $\left(I_{\min }\right)$ and white $\left(I_{\max }\right)$ areas of original image. For the original image $V_{0}=1$, because $I_{\max }=1$ and $I_{\min }=0$ and $V=0$ for uniformly painted image. For the restored image it decreases as the masking level increases. The second experiment was carried out with the image composed of equal number of black and white squares placed in fixed positions in random order (Fig. 2b). The image was representing a set of 1024 data bits, and the measured parameter was the bit detection accuracy (BDA) for the restored image

$$
D=\frac{T-F}{T+F}
$$

where $T$ (true) is the number of correctly detected bits and $F$ (false) is the number of incorrectly detected bits. It is clear that in this case always $T+F=1024$. Bit detection procedure is the following:

1) obtaining the average intensity over whole image $I_{\mathrm{A}}$;

2) averaging the intensity in the output image over every fixed square area representing single bit;

3) threshold the intensity of every single bit area by $I_{\mathrm{A}}$;

4) read the bits states.

$D=1$ for all the bits detected correctly and $D=0$ for the case of stochastic detection, when only a half of the bits is detected correctly just by chance. For the restored image it decreases as the masking level increases. 


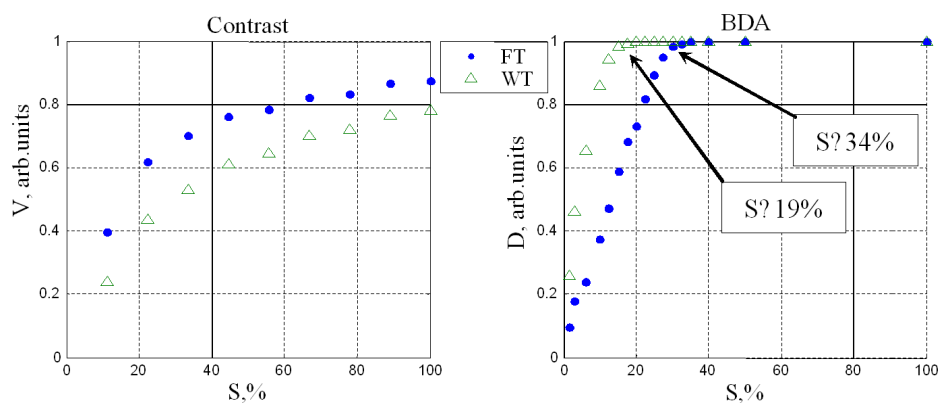

Fig. 3. The results of the experiments for contrast (a) and bit detection accuracy (b). $V$ - mean contrast defined by (7); $D$ - bit detection accuracy defined by (8); $S$ open area of the reconstructing image.

The output results of the experiments are the dependences of $V$ and $D$ against the open area $S$ of the restoring image. Corresponding plots are shown in Fig. 3a $(V)$ and Fig. 3b $(D)$.

\section{Conclusions}

As seen from the results (Fig. 3a,b) our VO scheme works better in contrast test exploiting FT while WT works better in bit detection test. That means that FT produces subjectively better looking image with less amount of noise whereas WT produces images with more homogeneous noise, which is obviously more important in task like bit detection.

From Fig. 3b it is clear that the dependence $D(S)$ begins with almost linear rapid rise up, reaches the value of 1 and then does not change. At that such dependence may be characterized with just one parameter. Let us call it the fallout point. Thus we may state that our VO scheme based on FT has a fallout point at $34 \%$ of open area of restoring image. And the WT-based scheme has a fallout point at $19 \%$, which is significantly better than the FT case.

\section{References}

[1] X. Peng, Z. Cui, T. Tan, Opt. Commun. 212, 235 (2002).

[2] X. Wang, D. Zhao, Opt. Express 14, 1476 (2006).

[3] B.M. Hennelly, J.T. Sheridan, Optik 114, 251 (2003).

[4] J. Garcia, D. Mas, R.G. Dorsch, Appl. Opt. 35, 7013 (1996).

[5] http://bsfp.media-security.ru/school6/5.htm.

[6] A. Gnatovsky, O. Zolochevska, N. Kukhtarev, Optical Memory Neural Networks 7, 107 (1998).

[7] A. Derzhypolskyy, D. Melenevskyy, A. Gnatovskiy, N. Medved, Funct. Mater. 12, 1 (2005).

[8] http://www.icaen.uiowa.edu/ dip/LECTURE/LinTransforms.html. 